between events recorded independently by these two experiments that Weber puts forward as proof of the existence of gravitational waves, because there seems to be no other phenomenon which could disturb both bars in the appropriate way at the same time. Unfortunately, however, many astronomers are concerned about the statistical techniques used by Weber in deciding which coincidences are real.

But just why do Weber's claims cause so much concern? The answer is simply that they seem too much of a good thing. Dr M. J. Rees (University of Cambridge) explained just what the observations imply, taken at face value. Weber reports bursts of gravitational waves, coming roughly from the centre of our galaxy at a rate of one or two per day. If this is typical, and the waves are emitted isotropically from the galactic nucleus (some $10 \mathrm{kpc}$ from Earth), then the luminosity of the nucleus in gravitational radiation alone is $10^{51} \mathrm{erg} \mathrm{s}^{-1}$. Although it is now known that in certain circumstances the collision of two black holes can result in the loss of half the total mass of the pair as gravitational waves, the total mass loss from the galaxy implied by this large luminosity is $2 \times 10^{4} \mathrm{M}_{\odot} \mathrm{yr}^{-1}$. This is the figure on which many astronomers choke, for it implies that the galaxy is losing mass so rapidly that there should be observable effects on the orbits of its constituent stars. These effects are not seen.

There are two ways out of this dilemma. Either Weber is wrong or general relativity is wrong. Each alternative raises interesting possibilities. First, if Weber has not detected gravitational waves, what has he detected? Second, if general relativity can be proved to be wrong in this instance the gates will be opened, once and for all, to those theoreticians who have been claiming for some time that a new theory of gravitation is needed. One suggestion put forward at the meeting is that if matter creation is taking place at the nucleus of our galaxy then gravity waves might be produced in abundance by the same process.

These questions may now be resolved in a matter of months. Throughout the world, many groups are working on the search for gravitational waves, and the first Weber type detector in Britain has just been completed in Glasgow. The Bristol team also have detectors in an advanced stage of construction. A joint Bristol-Glasgow experiment seems particularly desirable, because not only would this have a suitably large baseline to remove spurious effects but also it would have the advantage of two independent teams with no personal axes to grind conducting the tests for genuine coincidences.

\title{
CHEMISTRY
}

\section{Probing the Structure of Acetylacetone}
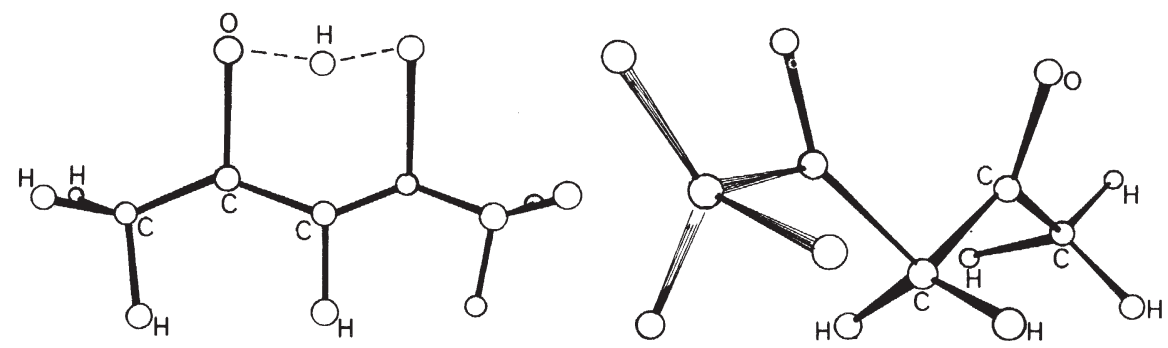

Fig. 1 Structures of enol and keto forms, respectively, of acetylacetone.

Electron diffraction studies of the "enol" form of acetylacetone-the tautomeric species that makes up varying proportions of the compound at different temperatures, 65 per cent at $100^{\circ} \mathrm{C}$ for example-have revealed that the hydrogen bond between the two oxygen atoms is linear, symmetric and short in the vapour phase.

Although this is not an entirely unexpected result in view of the discovery in 1966 of a similar short bond $(2.424$ $\AA)$ in tetraacetylethane, a dimer of acetylacetone, the even smaller value of the OO separation in acetylacetone(enol) of $2.381 \AA$ is somewhat surprising and indicates that the five-membered ring has definite aromatic characteristics.

These structural determinations are described by Lowrey, George, D'Antonio and Karle (J. Amer. Chem. Soc., 93, 6399 ; 1971). Fig. 1 shows, approximately to scale, the structures deduced by Lowrey et al. for both the keto and enol forms. The OO bond length of the keto form is $2.767 \AA$.

Acetylacetone is important because of its ability to undergo the chelation process, forming heterocyclic rings containing at least one metal cation or one hydrogen ion (as in the enol form). The metal chelate ring compound of the enol form of acetylacetone contains very stable six-membered rings, and the overall stability is reflected in the fact that beryllium acetylacetonate, $\mathrm{Be}(\mathrm{acac})_{2}$, boils without decomposition at $270^{\circ} \mathrm{C}$.

\section{Silicute Liquid Immiscibility}

Although molten rock erupted at the surface and intruded into the Earth's crust is essentially a silicate melt, it displays wide variations in its chemistry, the elucidation of which has long taxed the ingenuity of petrologists. By far the most popular explanation is that most igneous rocks start as low-silica, highmagnesia melts in the Earth's mantle, which are then modified on their way to the surface by processes of crystallization or contamination. Another possibility is that primitive melts might separate on cooling into two or more immiscible liquids, to provide a range of lava compositions. The few adherents to this idea had previously to contend with abundant experimental evidence against liquid immiscibility in those silicate melts which correspond to common rock compositions. Evidence of liquid immiscibility in the glassy parts of the lunar samples from Apollo 11 , however, has undoubtedly helped to stimulate renewed interest in terrestrial examples, as witnessed in recent geological literature (see, for example, Nature, 229, 107 ; 1971).

In next Monday's Nature Physical Science (January 31) another example of immiscibility is claimed by $\mathbf{J}$. Ferguson and $\mathrm{K}$. $\mathrm{L}$. Currie to be present in some of the oldest volcanic rocks of the Earth's crust, the Swaziland Sequence of southern Africa. Some of the dark basaltic lavas contain pale globules up to $10 \mathrm{~cm}$ in diameter which, the authors argue, formed as immiscible drops from an originally homogeneous liquid as it was cooled. Melting experiments on these lavas show signs of liquid unmixing between $1,100^{\circ}$ and $1,200^{\circ} \mathrm{C}$.

Analysis of the two components of the globular rocks shows a spread of composition that could explain part of the total chemical range of the Swaziland lavas: Ferguson and Currie deduce that liquid unmixing was an important factor in producing this range of compositions. The ancient Swaziland rocks are now wholly crystalline and, moreover, have suffered mineralogical alteration; some petrologists will argue that, consequently, their precise liquid compositions are unknown and that the phase structure cannot be accurately recreated.

Many other questions will be raised by Ferguson and Currie's conclusion, not least of which will be the longstanding fundamental problem of how two silicate liquids could be cleanly separated to produce distinct eruptions. 